\section{THE POULSEN SELECTIVE SYSTEM OF WIRELESS TELEGRAPHY.}

\section{BY A. FREDERTCK COLLINS.}

A new system of wireless telegraphy that gives considerable promise of solving the extremely difficult problem of selectivity, i. e., the transmission and reception of a number of messages in the same field of force simultaneously and without interference, has been devised and tried out by Valdemar Poulsen, the well-known inventor of the telegraphone.

The arrangement by which Poulsen hopes to accomplish the paramount result that is summed up in the word selectivity consists essentially of a new type of generator for producing continuous electrical oscillations. This generator will be subsequently described in detail, and the advantage of such high frequency cur rents over those having a periodic character that have hitherto been employed will also be pointed out.

Ever since 1897, when Sir Oliver Lodge applied to wireless telegraph transmitters and receptors the combination of open and closed circuits, and introduced the methods of tuning the circuits at either station individually and syntonizing them collectively, have persistent efforts been made by physicists and other to secure a suitable degree of resonance by providing the proper values of inductance, capacity, and resistance, and when these conditions prevailed, it was concluded the receiving resonator system would respond to a specific radiating oscillator system and to this one only. It is a matter of common knowlenge that these tuning effects have been brought to a highly perfected state, thanks to the development of instruments which enable the operator easily to determine the inductance and capacity values, the frequency of the oscillations and the length of the emitted waves to be measured. It is likewise well known that syntonization as it has been practise has not fulfille the requirements of selectivity in its commercial as pects, although it works out admirably from a theoretical viewpoint.

Nevertheless, these tuned and syntonized systems have served their purpose in extending the signaling distance as well as in reducing the initial energy but the fact that the oscillations set up were periodic, and that between each succeeding train of oscillations a long interval of time elapses-that is, long com pared with the time require for the oscillations to surge through the system before they. are damped out into the form of electric waves-has militated against their usefulness as a means to the end of securing selectivity.

Believing that continuous oscillations would remedy this inherent defect Poulsen employs for the purpose the musical arc, whose phenomena were discovered by Duddell in 1900. The apparatus for producing the arc consists of an arc light, fed by a direct current, around which is shunted a condenser in series with an inductance coil as shown in Fig. 2, in which $A$ is a chokductance coil as shown in Fig. 2 , in which $A$ is a chok
ing coil, $B$ the inductance coil, $C$ the condenser in the shunt circuit, ${ }^{-} D$ a regulating resistance inserted in the direct-current circuit, and $X$ the arc light. Now when certain conditions are satisfied the arc will give out a musical note and at the same time it will trans form a portion of the direct current into an alternating current the amplitude of which is constant; and the energy dissipated in the condenser circuit in the form of ohmic losses is supplied by the direct cur rent

Duddell ascertained, however, that the arc light will emit a musical note only when there is a small change in the potential difference between the electrodes of the arc, and when the corresponding change in the current through the arc is numerically greater than the resistance in the condenser, and numerically less than the resistance of the direct-current circuit in series with the arc. These conditions are fulfilled if the arc is formed between solid carbons. This is an exceedingly simple method of producing not. only high method of producing not. only high-
frequency currents but also a very easy way of setting up continuous electric oscillations.

When experimenting some years ago with the musical arc, Poulsen made an observation which he followed up by occasional experiments, and which finally ied him to the construction of a generator for producing continuous electric oscillations. In his first apparatus the carbons were placed horizontally and co-axially, as shown in Fig. 4, thus permitting an ordinary alcohol lamp to be held under the arc in such a manner that the latter and adjacent parts of the electrodes were surrounded by the vapor from the alcohol. An ordinary ammeter, $A$, was placed in the direct current circuit, and the hot-wire ammeter, $B$, for measuring high-frequency alternating currents, was place in the condenser circuit. The direct current was taken from a 220 -volt lighting cir. cuit. Now, when the arc light was placed in the spirit vapor as cited above, a musical note was emitted, the pitch of which diminished. At the same time the hot-wire ammeter indicated a considerable increase in the oscillating current, while the direct current decreased in value. The result was further accentuate by the arc light's glowing less brightly. In succeeding experiments hydrogen and hydrogen com

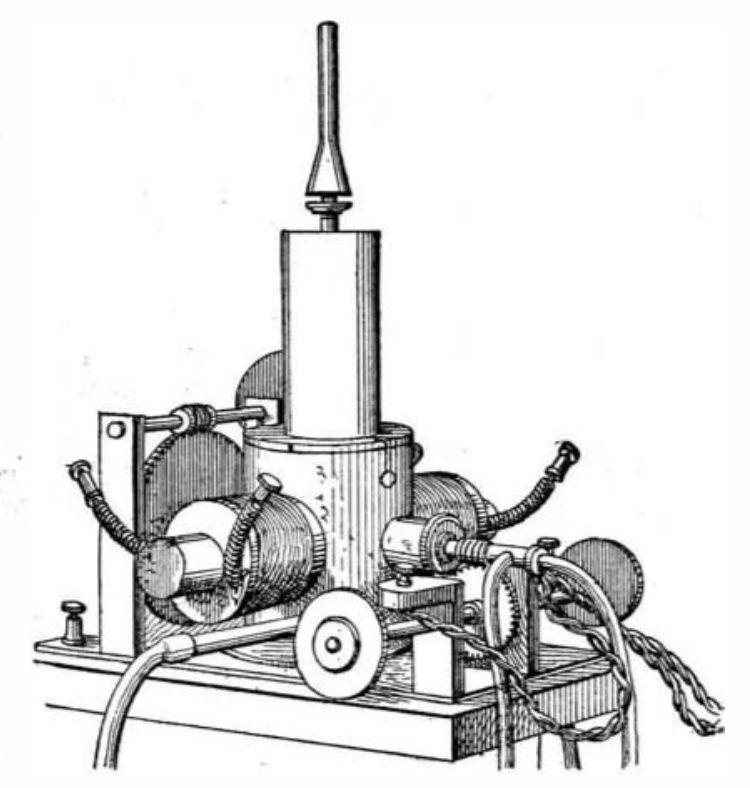

Fig. 1.-Poulsen Hydrogenic Arc Emitter.

pounds were used with approximately similar results, the length of arc required to give a maximum current in the condenser circuit being different than it was those cases in which the arc was formed in air.

When copper and charcoal were employed as the electrodes, and a nitrogen compound was drawn through the walls of the carbon into the arc, where it was vaporized, the strongest continuous oscillations of

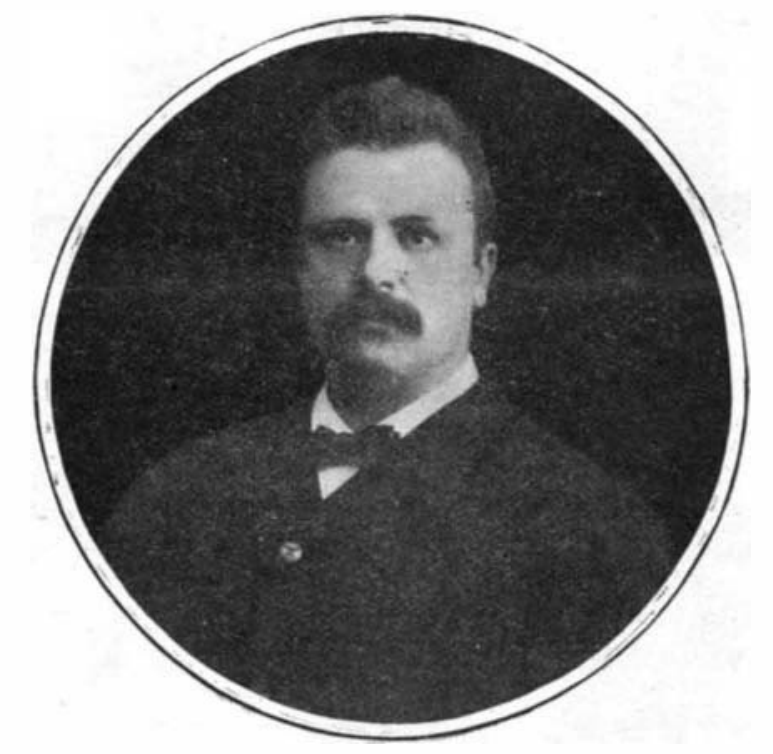

VALDEMAR POULSEN.

constant amplitude were obtained, and these currents were further reinforced by blowing the arc out to a greater length with an electro-magnet, thus increasing its resistance. This is what the inventor terms a hydrogenic arc, and when its image was projected upon a screen with the condenser circuit broken, the arc was observed as a greenish blue spot having a faint core of a purple tint. When the arc was made musical

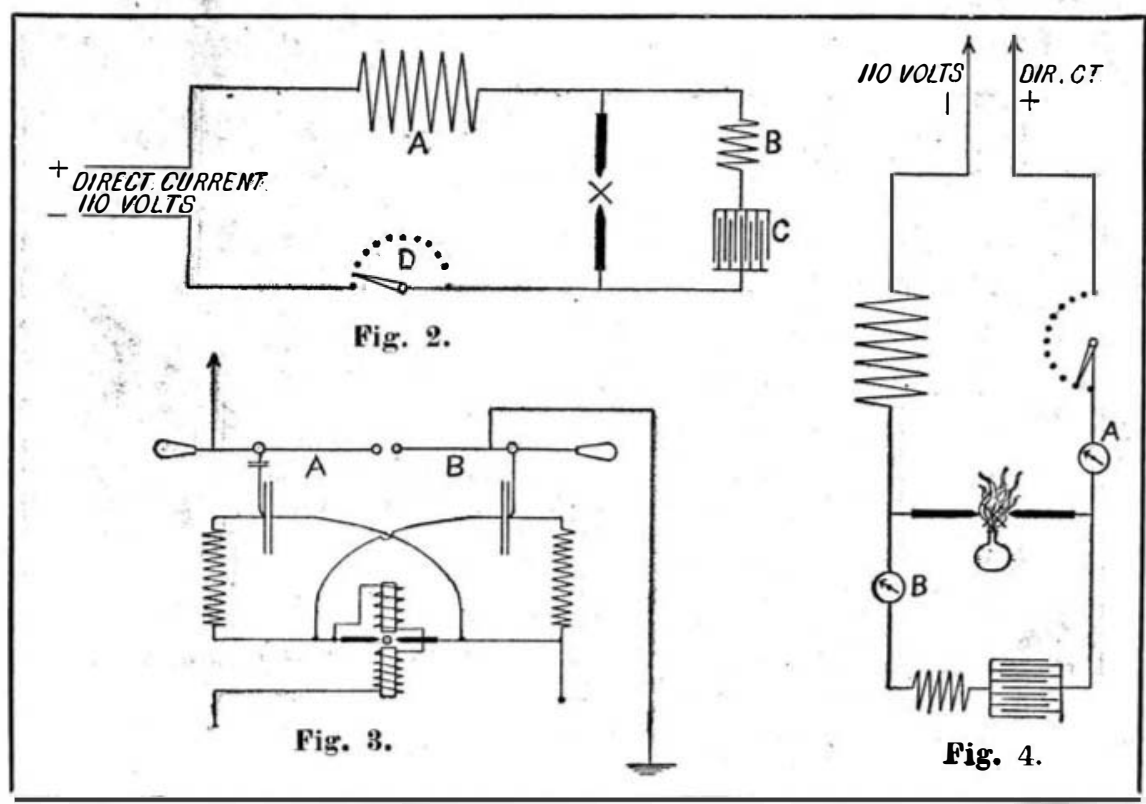

POULSEN'S SELECTIVE SYSTEM OF WIRELESS TELEGRAPHY, by closing the condenser circuit, the colored spot became deeper and the purple core was very much more marked. This hydrogenic arc gave out musical notes, or rather electrical notes, the inventor tells us, of several hundred thousand oscillations per second, but it is possible to obtain some millions of oscillations per second. The excellent resonant effects that can be obtained with these oscillations indicate their continuity, and in a rotating mirror it will be seen that the oscillations are actually continuous.

With currents of high frequency spirits did not give as good results as hydrogen, nitrogen, coal gas, or ether. Again, it was shown to be necessary to draw the arc out to a certain length in order to start the oscillations, but when these have been started the length of the arc can generally be cut down a trifle without causing them to cease. If the length of the arc is increased, then the oscillations will continue, and will cease only when the distance between the electrodes has become'so great that the arc is extinguished.

In some of his experiments Poulsen obtained in the condenser circuit-that is, the circuit in which the oscillations are set up-energy equivalent to about 1,560 watts (a little over 2 horse-power) the arc at the same time taking from the direct circuit about 3,170 watts, the efficiency thus being about 50 per cent In these tests no effort was made to obtain the greatest efficiency or effect, the arc being placed in a watercooled vessel without an outlet for the gas, this ar. rangement being necessary to determine the amoun of energy consumed and transformed. It also les sened the intensity of the oscillations, since the composition of the gas was altered.

The Poulsen system of producing continuous electric oscillations is capable of taking care of a considerable amount of energy even when very high frequencies are used, as has been repeatedly proven by different experiments in connection with the ordinary lighting circuit of 220 volts. For instance, a resonating coil gave, when inserted in a properly balanced oscillating circuit, a noiseless, warm flame having a length of 12 centimeters; and if the flame is made short, it can be easily observed to be continuously oscillating, by means of a rotating mirror.

Another test was made by placing a large X-ray tube between the coils inductively coupled with an oscillation circuit, and in a short time the cathode and anti-cathode melted. Again, an ordinary 200-volt incandescent lamp glowe when placed in series with two persons, one of whom was connected with the oscillating circuit. Further, if the secondary coil of an ordinary induction coil is surrounded by a helix of thick copper wire, and the terminals are connected in series with a capacity of some microfarads capacity, the shunting in of the nitrogenic arc will produce a very loud singing flame 10 or 12 centimeters in length or more, and finally, an X-ray tube with this arrangement gives a very strong radiation.

From the foregoing it is obvious that such continuous electric oscillations are eminently adapted to the production and propagation of electric waves for wireless telegraphy, and that by properly proportioning the radiating aerial and receiving antenna the desire wave length can be emitte and utilize to produce a continuous resonance effect. The diagram, Fig. 3, shows two oscillating circuits tuned to the same frequency, and by means of which oscillating flames of about double the voltage can be obtained. To provide a wireless telegraph transmitter, it is neces sary only to connect the radiating aerial wire with one side of the spark-gap $A$ and the earthed terminal to the opposite side of the gap $B$.

The receptor may have any type of detector, al though the magnetic detector is exceptionally well adapted for selective signaling in connection with the Poulsen hydrogenic arc emitter, the latter being shown in the illustration, Fig. 1. It is stated on good authority that 1. It is stated on good authority that
a dozen messages have been transmitted and received between as many experimental sets by means of this new se lective system without interference; and if this extraordinary result can be duplicated over distances of 50 or 100 miles, as the experiments thus far made between the inventor's two Danish stations indicate, an advance will have been made that, in its importance, will be second only to the introduction of wireless telegraphy itself.

For cleaning pivot holes in small machinery, use a small soft stick, tapering so as to enter easily, but fitting tightly in the hole. This is put in and twirled around, and driven through "until the hole no longer dirties it, when the hole may be pronounced clean, although a little petroleum, or better yet, benzine, may be use to "make assurance doubly sure." 